\title{
Separation of Aromatic Isomers by Capillary Gas Chromatography with Two Calix[4]arene Polysiloxane Stationary Phases
}

\author{
Jun XING, Cai-Ying Wü, Tao LI, Zhen-Lin Zhong and Yuan-Yin Chen \\ Department of Chemistry, Wuhan University, Wuhan 430072, P. R. China
}

\begin{abstract}
Two new stationary phases, cone bis(undecenyloxy)-p-t-butyl calix[4]crown-5 polysiloxanes (PSO-C-c[4]c-5) and partial cone 5,11,17,23-t-butyl-25,27-diethoxy-26,28-diundecenyl calix[4]arene polysiloxane (PSO-PC-c[4]E), are synthesized and used as stationary phases in capillary column gas chromatography. Their chromatographic characteristics, such as column efficiency, capacity factor, average polarity, operation temperature range, and peak asymmetry were measured. Their column efficiency is over 3000 plates $\mathrm{m}^{-1}$, and their average polarities are between that of OV-1701 and SE-54. Their maximum allowable operating temperature is up to $310^{\circ} \mathrm{C}$. Then, 33 aromatic compounds and positional isomers were used to test the separation abilities of the two stationary phases. The results indicate that the synthetic calixarene stationary possess good separation ability for aromatic compounds and positional isomers. It is noticeable that the retention behavior of several positional isomers on the PSO-C-c[4]c-5 column are different from that on PSO-PC-c[4]E column. The separation mechanism is also discussed.
\end{abstract}

Keywords Calixarene siloxane telomer, stationary phases, separation, aromatic isomers, gas chromatography

Calixarenes, macrocyclic phenol-formaldehyde polycondensates, have become significant in supramolecular chemistry during the past two decades. Their configuration is very like a chalie or a cup and so they are named calixarene. The origin of calixarene can be traced back to Von Baeyer's discovery of phenolformaldehyde resins in the $1870 \mathrm{~s}$. Since then, many members of the series from $n=4-14$ are known. Because of its basket-shaped cavity, it can form complexes with neutral molecules ${ }^{1}$ and ions. ${ }^{2-4}$ There have been many papers on synthesis of calixarene ${ }^{5-7}$, modification of its upper ${ }^{8}$ and lower rims ${ }^{9}$, and applications, such as using them as accelerators for instant adhesives ${ }^{10}$, stationary phases for chromatography, ion selective electrodes and field effect transistors ${ }^{11}$ and ion scavengers for electronic devices ${ }^{12}$, and to purify fullerenes. ${ }^{13}$

Our group has worked on stationary phases containing crown ethers in gas chromatography for many years. However, ordinary crown ether is a plane molecule with a two-dimensional structure. If a molecule with a three-dimensional structure such as calixarene takes the place of crown ether, the recognition and separation ability of stationary phase seems to be improved. Feltl, et al. firstly used the basic, unsubstituted calixarene as stationary phases in gas chromatography ${ }^{14,15}$, but the result indicated low column efficiency. It can be attributed to the poor solubility and high melting point of calixarene. Hence, the utility of calixarene in separations was greatly restrained. Derivatization being a practicable method to improve the solubility, a few reports are available using modified calixarene as stationary phase in gas chromatography. ${ }^{16}$

In this paper, two new telomers, containing respectively cone bis(undecenyloxy)-p-t-butyl calix[4]crown-5 poly siloxanes (PSO-C-c[4]c-5) and partial cone 5,11,17,23-t-butyl-25,27-diethoxy-26,28-diundecenyl calix[4]arene polysiloxane (PSO-PC-c[4]E), are synthesized in a new way and used as stationary phase for capillary gas chromatography. The new stationary phases exhibit excellent column efficiency, thermal stability and unique selectivity in separating many aromatic compounds, especially for positional isomers.

\section{Experimental}

\section{Synthesis of the two siloxane telomers}

The calixarene derivatives, bis(undecenyloxy)- $p$ - $t$ butyl calix[4]crown-5 and 5,11,17,23-t-butyl-25,27diethoxy-26,28-diundecenyl calix[4]arene were supplied by Dr. Zhong. Both calixcrown polysiloxane, PSO-C-c[4]c-5 (I) and PSO-PC-c[4]E (II), were prepared as the procedures in ref. 17.

Their structures were characterized by IR and ${ }^{1} \mathrm{H}$ NMR spectra and elemental analyses. The conformations of calixarene units in PSO-C-c[4]c-5 and PSOPC-c[4]E, which was deduced from their ${ }^{1} \mathrm{H}$ NMR spectra and structures of their monomers, were cone and partial cone respectively (see Fig. 1).

† To whom correspondence should be addressed. 


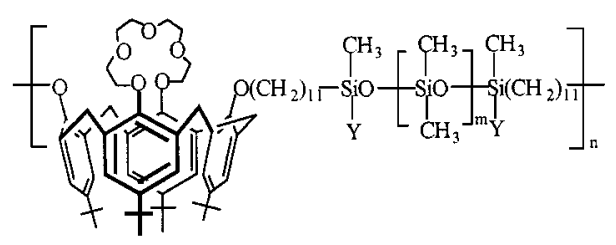

PSO-C-c[4]c-5

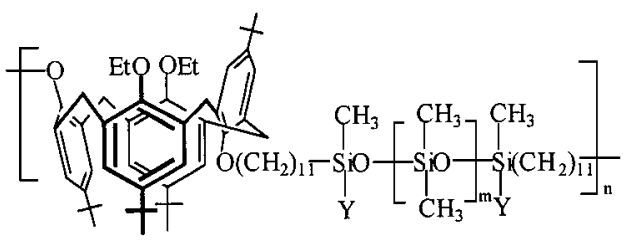

PSO-PC-c[4]E

Fig. 1 Structures of PSO-C-c[4]c-5 and PSO-PC-c[4]E.

Table 1 Characteristics of PSO-C-c[4]c-5 and PSO-PC-c[4]E capillary columns

\begin{tabular}{ccccccc}
\hline $\begin{array}{c}\text { Stationary } \\
\text { phase }\end{array}$ & $\begin{array}{c}\text { Column } \\
\text { No. }\end{array}$ & $\begin{array}{c}\text { Column efficiency } \\
\text { (plates/m) }\end{array}$ & $\begin{array}{c}\text { Naphthalene } \\
\text { capacity factor }\left(k^{\prime}\right)\end{array}$ & Av. polarity & $\begin{array}{c}\text { Operating temperature } \\
\text { range/ }{ }^{\circ} \mathrm{C}\end{array}$ & Peak asymmetry \\
\hline \multirow{2}{*}{ PSO-C-c[4]c-5 } & 1 & 4000 & 3.51 & 87 & $110-310$ & 1.08 \\
& $1^{\prime}$ & 4166 & 3.42 & 88 & & 1.06 \\
PSO-PC-c[4]E & 2 & 3333 & 3.50 & 75 & $100-310$ & 1.03 \\
& $2^{\prime}$ & 3134 & 3.60 & 73 & & 1.01 \\
\hline
\end{tabular}

\section{Apparatus}

A GC-7A gas chromatograph (Shimadzu, Japan) equipped with a capillary split injection system and flame ionization detector was used throughout. Nitrogen was used as carrier gas and the split ratio is $1 / 50$. The injector and detector temperatures were maintained at $250^{\circ} \mathrm{C}$ and $280^{\circ} \mathrm{C}$, respectively. Peak measurement and integration were performed by a CR3A chromatographic recorder. The length and inner diameter of capillary column is $20 \mathrm{~m}$ and $0.25 \mathrm{~mm}$ respectively.

\section{Capillary column preparation}

Fused-silica capillary columns (YongNian optical fiber factory, Hebei, P. R. China) were rinsed with 10 $\mathrm{ml}$ of methanol and purged with Nitrogen at $250^{\circ} \mathrm{C}$ for $2 \mathrm{~h}$ to remove acidic impurities. The columns were then statically coated with a solution of $0.5 \%(\mathrm{w} / \mathrm{v})$ PSO-C-c[4]c5 and PSO-PC-c[4]E in dichloromethane at $30^{\circ} \mathrm{C}$. Following the coating and flushing with nitrogen for $3 \mathrm{~h}$, the columns were conditioned at $280^{\circ} \mathrm{C}$ for $12 \mathrm{~h}$ under a slow flow of nitrogen before use. The column coated with PSO-C-c[4]c5 was column 1, and the other coated with PSO-PC-c[4]E was column 2. For testing the repetition of the new stationary phases, columns $1^{\prime}$ and $2^{\prime}$ were prepared, which were coated respectively with PSO-C-c[4]c-5 and PSO-PC-c[4]E synthesized another time. The characteristics of the columns, such as column efficiency, glass transition temperature, polarity, selectivity of isomer separation were tested.

\section{Results and Discussion}

Chromatographic characteristic

Both PSO-C-c[4]c5 and PSO-PC-c[4]E contain the nonpolar polysiloxane main chains and macrocycles, they are predicted to possess good film-forming ability and high thermal stability and unique selectivity for aromatic compounds. Table 1 shows some properties of these two calix[4]arene capillary columns, such as column efficiency, peak asymmetry factors, average polarity, and operating temperature range.

The data in Table 1 indicate that the efficiencies of the two columns are over 3000 plates $\mathrm{m}^{-1}$, which is much higher than those of stationary phases containing basic calixarene, and their reproducibility are good. The peak asymmetry factors for 1-octanol on the fusedsilica columns are close to 1.0 , such a result exhibits the good film-forming ability of calix[4]arene siloxane telomers. The average polarity of two stationary phases, which were expressed by McReynolds constants measured at $120^{\circ} \mathrm{C}$, showed that the two phases exhibit weak polarity, and their polarity are between OV-1701 and SE-54. The polar crown ether ring in PSO-C-c[4]c-5 accounts for the difference in polarity between them.

The operating temperature range in Table 1 is determined by the glass transition temperature and column bleeding. The plots of $\log k^{\prime}$ of naphthalene versus reciprocal absolute temperature for the two columns were drawn. The changes in slope at $110^{\circ} \mathrm{C}$ and $100^{\circ} \mathrm{C}$ correspond to glass transition points for column 1 and column 2 respectively. The column bleeding was measured by programming columns from $140^{\circ} \mathrm{C}$ to $310^{\circ} \mathrm{C}$ at $4^{\circ} \mathrm{C} \mathrm{min}{ }^{-1}$. The results show that columns 1 and 2 began to bleed at the same temperature $270^{\circ} \mathrm{C}$, baseline shifts were $5.2 \times 10^{-14}$ and $6.0 \times 10^{-14} \mathrm{~A}$ at $310^{\circ} \mathrm{C}$ respectively. This demonstrated their high thermal stability.

Grob test mixtures were used to evaluate the overall chromatographic properties of the capillary columns. Figure 2 shows that the mixtures were well separated 


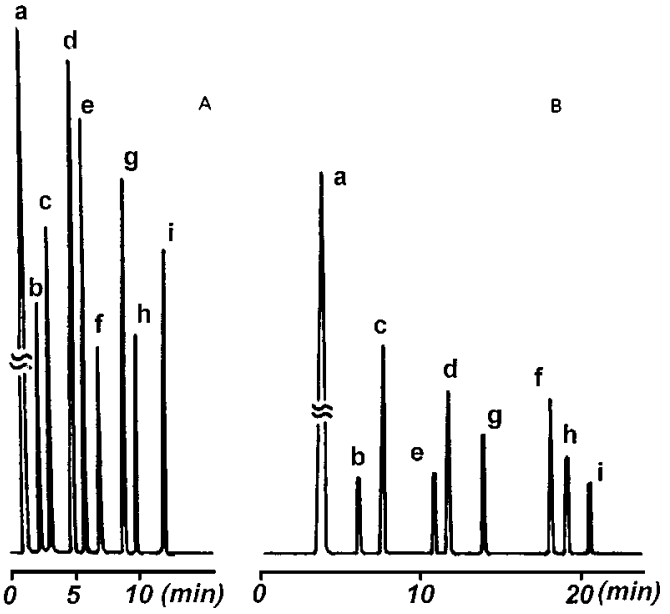

Fig. 2 Chromatogram of Grob test mixture on column 1(A) and column 2 (B). Temperature: $140^{\circ} \mathrm{C}$. a, solvent; b, 1,3butanediol; c, decane; d, undecane; e, octanol; f, dodecane; g, 2,6-dimethylphenol; h, 2,4-dimethylaniline; i, naphthalene.

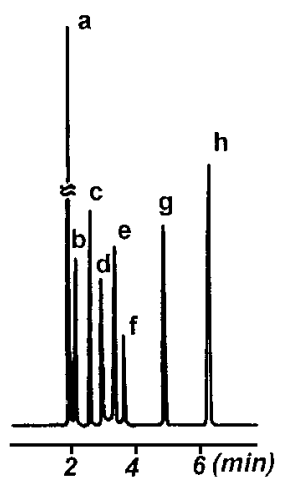

Fig. 3 Chromatogram of aromatic compounds on column 1. a, solvent; b, benzene; c, methylbenzene; d, o-xylene; e, $p$ xylene; f, $m$-xylene; g, 1,2,3-trimethylbenzene; h, 1,3,5trimethylbenzene.

and gave symmetrical peaks. It is notable that: 1octanol eluted behind undecane in column 1; but in column 2 the orders reversed. That indicated the hydrogen-bonding interaction between crown ether ring and the alcohols. For the same reason, 2,6-dimethylphenol eluted behind undecane in column 1 , but before in column 2.

\section{Separation of positional isomers}

We have studied the separation of some aromatic positional isomers on the two stationary phases. Table 2 shows the data of capacity factors $\left(k^{\prime}\right)$ and separation factors $(\alpha) . \alpha$ is the ratio of the capacity factors of two isomers, that indicates the separation ability of stationary phase for positional isomers. Figure 3 shows the chromatogram of the mixture of seven aromatic compounds on column 1 at $80^{\circ} \mathrm{C}$. It can be seen that column 1 gives the base-line separation for the mixture of seven aromatic compounds and aromatic positional iso-
Table 2 Retention data for aromatic isomers on different columns

\begin{tabular}{|c|c|c|c|c|c|c|}
\hline \multirow{2}{*}{ Compound } & & \multicolumn{2}{|c|}{ Column 1} & \multicolumn{2}{|c|}{ Column 2} & \multirow{2}{*}{$\begin{array}{l}\text { Column } \\
\text { temp. } /{ }^{\circ} \mathrm{C}\end{array}$} \\
\hline & & $k^{\prime}$ & $\alpha$ & $k^{\prime}$ & $\alpha$ & \\
\hline \multirow[t]{3}{*}{ Dimethylbenzene } & $o$ & 1.10 & 1.00 & 1.32 & 1.00 & 80 \\
\hline & $m$ & 1.89 & 1.72 & 1.72 & 1.30 & \\
\hline & $p$ & 1.24 & 1.13 & 1.44 & 1.09 & \\
\hline \multirow[t]{3}{*}{ Dichlorobenzene } & $o$ & 2.31 & 1.22 & 2.03 & 1.00 & 100 \\
\hline & $m$ & 1.90 & 1.00 & 2.44 & 1.20 & \\
\hline & $p$ & 1.98 & 1.04 & 2.13 & 1.05 & \\
\hline \multirow[t]{3}{*}{ Nitrotoluene } & $o$ & 2.43 & 1.10 & 2.17 & 1.08 & 140 \\
\hline & $m$ & 2.21 & 1.00 & 2.00 & 1.00 & \\
\hline & $p$ & 2.43 & 1.10 & 2.17 & 1.08 & \\
\hline \multirow[t]{3}{*}{ Chlorophenol } & $o$ & 0.28 & 1.00 & 0.71 & 3.22 & 170 \\
\hline & $m$ & 0.78 & 2.76 & 0.22 & 1.00 & \\
\hline & $p$ & 0.97 & 3.43 & 0.27 & 1.21 & \\
\hline \multirow[t]{3}{*}{ Dinitrobenzene } & $o$ & 2.28 & 1.00 & 2.11 & 1.00 & 170 \\
\hline & $m$ & 2.47 & 1.08 & 2.23 & 1.06 & \\
\hline & $p$ & 3.08 & 1.35 & 2.38 & 1.13 & \\
\hline Quinoline & & 0.99 & 1.00 & 1.05 & 1.00 & 170 \\
\hline Isoquinoline & & 1.21 & 1.23 & 1.38 & 1.31 & \\
\hline \multirow[t]{2}{*}{ Methylnaphthalene } & $\alpha$ & 1.31 & 1.25 & 1.25 & 1.00 & 170 \\
\hline & $\beta$ & 1.05 & 1.00 & 1.37 & 1.10 & \\
\hline Phenanthrene & & 7.96 & 1.00 & 7.73 & 1.00 & 180 \\
\hline Anthracene & & 8.29 & 1.04 & 8.08 & 1.05 & \\
\hline \multirow[t]{3}{*}{ Nitrochlorobenozene } & $o$ & 2.17 & 1.09 & 2.14 & 1.08 & 140 \\
\hline & $m$ & 1.99 & 1.00 & 1.98 & 1.00 & \\
\hline & $p$ & 2.17 & 1.09 & 2.14 & 1.08 & \\
\hline \multirow[t]{3}{*}{ Benzenediol } & $o$ & 0.46 & 1.00 & 0.57 & 1.35 & 190 \\
\hline & $m$ & 0.94 & 2.03 & 0.42 & 1.00 & \\
\hline & $p$ & 1.61 & 3.49 & 0.60 & 1.43 & \\
\hline \multirow[t]{6}{*}{ Dinitrotoluene } & $3,4-$ & 0.57 & 1.00 & 0.59 & 1.00 & 170 \\
\hline & $2,3-$ & 0.78 & 1.36 & 0.78 & 1.32 & \\
\hline & $2,6-$ & 2.35 & 4.11 & 2.26 & 3.84 & \\
\hline & $2,5-$ & 2.93 & 5.11 & 2.78 & 4.73 & \\
\hline & $2,4-$ & 3.52 & 6.14 & 3.28 & 5.58 & \\
\hline & $3,5-$ & 3.89 & 6.87 & 3.58 & 6.09 & \\
\hline
\end{tabular}

mers. The data of $\alpha$ in Table 2 and Figs. 3 and 4 demonstrate that column 1 and 2 have unique selectivity for aromatic compounds and their positional isomers. Figure 4 shows the chromatogram of xylene isomers on column 1 and 2. It can be seen that xylene isomers were separated well. However, $o-/ m$-xylene usually can not be resoluted on most crown ether stationary phases. This advantage of calixarene stationary phase may be attributed to the three-dimensional steric structure of calixarene.

The data of $\alpha$ in Table 2 also indicated that on column 1 the elution order of positional isomers, such as dimethylbezene, dichlorobezene, methylnaphthalene, chlorophenol and benzenediol, is noticeably different from that on column 2. This may be attributed to the different conformation of calixarene in the two station- 


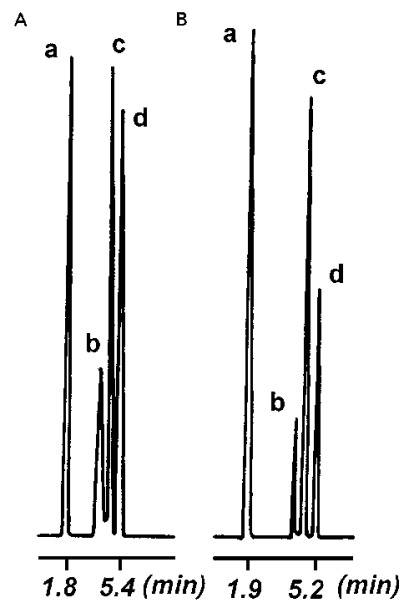

Fig. 4 Chromatogram of xylene isomers on column 1 (A) and column 2 (B). Column temperature, $100^{\circ} \mathrm{C}$. a, solvent; b, oxylene; c, $m$-xylene; $\mathrm{d}, p$-xylene.

ary phases. In addition to these, the data of $k^{\prime}$ in Table 2 and the retention time in Fig. 2 also indicate that the nonpolar and weak polar solutes were retarded longer on column 2 than on column 1 . This result may due to the different conformation of calixarene in the two stationary phases. In column 2 , because the conformation of calixarene is partial cone, the solutes entered the cavity of calixarene more easily. Also because $\mathrm{CH}$ (guest) $-\pi$ (host) interaction is the main force between nonpolar or weak polar solutes and calixarene, the retention time of alkane and alkyl aromatic compounds are longer in column 2 than in column 1.

\section{Separation mechanism}

As shown in Table 2, almost all aromatic positional isomers can be separated well except isomers of nitrotoluene and nitrochlorbenzene. Comparing $\alpha$ of the new stationary phases with that ${ }^{18}$ of $\operatorname{di}(t$-butylbenzo)propyl-15-crown-5 polysiloxane and dibenzo-propyl15-crown-5 polysiloxane, though whose structures are similar in some degree, the new stationary phases are better in separating nonpolar and weak polar isomers than others. Nonpolar isomers, such as isomers of $o$ $/ m$ - $/ p$-dimethylbenzene, quinoline/isoquinoline, $\alpha / \beta$ methylnaphthalene, phenanthrene/anthracene, are separated better on columns containing calixarenes than on that containing crown ethers; for polar isomers, it is the reverse.

The dispersion interaction, $\pi$ (guest)- $\pi$ (host) interaction and hydrogen bonding effect the elution order seriously. It is interesting that on the two calixarene columns the elution order of dinitrotoluene (DNT) isomers is not only dependent on the dipole-dipole interaction, but also on their boiling-point. The sequence of the dipole-dipole force is 2,5 -DNT $<2,4$-DNT $<2,3$ DNT $<2,6$-DNT $<3,5$-DNT $<3,4-\mathrm{DNT}$, but the order of retention time $\left(T_{\mathrm{R}}\right)$ is $T_{\mathrm{R} 3,4-\mathrm{DNT}}<T_{\mathrm{R} 2,3-\mathrm{DNT}}<T_{\mathrm{R} 2,6-\mathrm{DNT}}<T_{\mathrm{R} 2,5-\mathrm{DNT}}$ $<T_{\mathrm{R} 2 \text {,4-DNT }}<T_{\mathrm{R} \text { 3,5-DNT. }}$. The order of retention time is generally in agreement with that of their boiling points

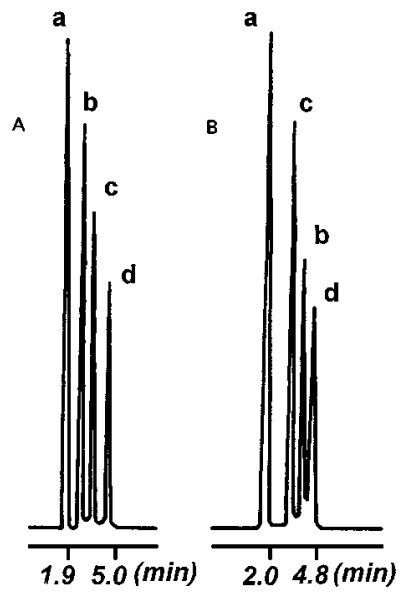

Fig. 5 Chromatogram of benzenediol isomers on column 1 (A) and column 2 (B). Column temperature, $190^{\circ} \mathrm{C}$. a, solvent; b, $o$-benzenediol; c, $m$-benzenediol; $\mathrm{d}, p$-benzenediol. except 2,5-DNT. This indicates that the dispersion interaction plays an important role in the separation of DNTs.

The two calix[4]arene columns also give good separation of xylene isomers which are generally difficult to separate by gas chromatography. Figure 4 shows the chromatogram of xylenes on the two columns. This can be attributed to the special $\mathrm{CH}$ (guest) $-\pi$ (host) and $\pi$ (guest) $-\pi$ (host) interaction between solutes and calixarenes.

Table 2 shows that chlorophenols and benzenediols were eluted in different sequences on column 1 and on column 2. Figure 5 exhibits the chromatograms of benzenediol isomers on the two columns. For column 1, the selectivity of phenol compound isomers depends on the availability of hydrogen bonding between the hydroxylic hydrogens and oxygen atoms in the crown ether residual. Because of the intramolecular hydrogen bonding in ortho-substituted phenols, which diminishes the hydrogen bonding between phenol and crown ether residual, the ortho-substituted phenols eluted earlier. For column 2, the selectivity may depend on dipole-dipole interaction.

The conformation of calixarene also influences the elution order of isomers. Comparing the elution order of dichlorobenzene and methylnaphthalene isomers in column 1 with that in column 2 , we can see that the elution order in column 1 is different from that in column 2. Possibly this is due to the different conformations of calixarenes.

In conclusion, PSO-C-c[4]c-5 and PSO-PC-c[4]E are two new weak polar stationary phases with high column efficiency possessing good film-forming ability and thermal stability. They exhibit excellent selectivity for aromatic positional isomers, especially for nonpolar and weak polar isomers. 


\section{References}

1. C. D. Gutsche and I. Alam, Tetrahedron, 44, 4689 (1988).

2. J. M. Harrowfield, W. R. Richmond and A. Sobolev, J. Chem. Soc., Perkin Trans. 1, 5 (1994).

3. J. Scheerder, M. Fochi and J. F. J. Engbersen, J. Org. Chem., 59, 7815 (1994)

4. F. Arnaud Neu, E. M. Collins, J. Am. Chem. Soc., 111, 8681 (1989).

5. C. D. Gutsche and M. Iqubal, Org. Synth., 68, 234 (1990).

6. C. D. Gutsche., B. Dhawan and M. Lenois, Org. Synth., 68, 238 (1990).

7. J. H.Munch and C. D. Gutsche, Org. Synth., 68, 243 (1990).

8. M. Almi, A. Aroluini and A. Casnati, Tetrahedron, $\mathbf{4 4}$ 4689 (1989).

9. Z.-T. Huang and G.-Q. Wang, Synth. Commun., 24, 11 (1994).

10. S. J. Harris, Europe Patent 151527 (1985).
11. D. Diamond and M. A. Mckervey, Chem. Soc. Rev., 1996, 15

12. R. Perrin, S. Harris, in "Calixarene, A Versatile Class of Macrocyclic Compounds", p. 235, ed. J. Vicens and V. Bohmer, Kluwer Academic Publishers, Dordrecht, 1991.

13. T. Suzuki, K. Nakashima and S. Shinkai, Chem. Lett., 1994, 699.

14. P. Munk and L. Feltl, J. Chromatogr., 696, 101 (1995).

15. P. Munk, L. Feltl and V. Shurig, J. Chromatogr., 732, 63 (1995).

16. L. Lin, C.-Y. Wu and Z.-Q. Yan, Chromatographia, 47, 689 (1998).

17. Z.-L. Zhong, C.-Y. Wu and Y.-Y. Chen, J. Chem. Soc., Chem. Commun., 16, 1737 (1995).

18. C.-Y. Wu, L.-S. Cai and Y.-J. Wang, Chromatographia, 37, 374 (1993)

(Received December 3, 1998) (Accepted April 26, 1999) 